\title{
First results from a beam test of a high-granularity silicon-based calorimeter for CMS at HL-LHC
}

Rajdeep M Chatterjee ${ }^{* \dagger}$

University of Minnesota, Minneapolis

E-mail: rmchatterjeejr@gmail.com

\begin{abstract}
A prototype of the electromagnetic calorimeter for the CMS High Granularity Calorimeter that is being designed for the High Luminosity LHC (HL-LHC) was tested at the Fermilab Test Beam Facility. The detector consisted of 16 sampling layers of silicon sensors interspersed with tungsten plates, for a total thickness of $15.3 \mathrm{X}_{0}$. Each of the hexagonal sensors were sub-divided into 128 cells, predominantly hexagonal in shape, of area $\sim 1.1 \mathrm{~cm}^{2}$. The analog signal from the 2048 cells was readout using the 64-channel SKIROC2 ASIC, developed by the OMEGA group for the CALICE collaboration. Data were collected with a custom data acquisition system developed for these tests. The detector was calibrated using signals obtained with $120 \mathrm{GeV}$ protons. We report here the design of the prototype detector and the results obtained from analyzing the data collected in July 2016, with electron beams at energies ranging from 4 to $32 \mathrm{GeV}$.
\end{abstract}

38th International Conference on High Energy Physics

3-10 August 2016

Chicago, USA

${ }^{*}$ Speaker.

${ }^{\dagger}$ On behalf of the CMS Collaboration. 


\section{Introduction}

The CMS collaboration has decided to replace the detector's endcap calorimeters with a new High Granularity Calorimeter (HGCAL) [1] for operations at the HL-LHC. This replacement is necessary because the calorimeters currently in place will not support the expected radiation fluence at the HL-LHC. For the new calorimeter the active material will be silicon sensors, that have been shown to be able to withstand the full dose and fluence expected[2]. The new calorimeter will be highly segmented both transversely and longitudinally throughout, which will enable event reconstruction using particle flow techniques [3]. It will built as three separate parts: a $25 \mathrm{X}_{0}$ electromagnetic section (EE) consisting of 28 silicon-tungsten sampling layers, a $3.5 \lambda$ front hadron calorimeter (FH) with silicon-steel sampling, and a $5.5 \lambda$ backing calorimeter, with steel as the absorber and scintillating plastic as the active medium. The EE and the FH sections will be maintained at $-30^{\circ} \mathrm{C}$, to minimize the effect of radiation on the silicon, and the present plan is to operate the $\mathrm{BH}$ at room temperature, but cold operation is also being explored.

\section{The HGCAL EE prototype}

As part of our program to qualify and optimize the calorimeter design, we have built and tested at the Fermilab Test BeamFacility (FTBF) a $15.3 \mathrm{X}_{0}, 16$-sampling layer prototype calorimeter. The sensors used for this test were made from 6" $p$-in-n silicon wafers manufactured by HPK ${ }^{1}$. The $320 \mu \mathrm{m}$ thick wafers had a depletion depth of $200 \mu \mathrm{m}$ and were segmented into 128 cells. The cells, apart from those on the periphery, were hexagonal with an area of $\sim 1.1 \mathrm{~cm}^{2}$.

In the construction of the prototype, the silicon sensors and readout electronics were assembled into detector modules. Each module consisted of a sintered W$\mathrm{Cu}(75 / 25)$ base plate, a metalized $\mathrm{KAPTON}^{\mathrm{TM}}$ sheet, the silicon sensor and two readout boards. A passive readout board was glued to the silicon sensor and electrical connection was made with through-hole wire bonds. This board was connected to a second

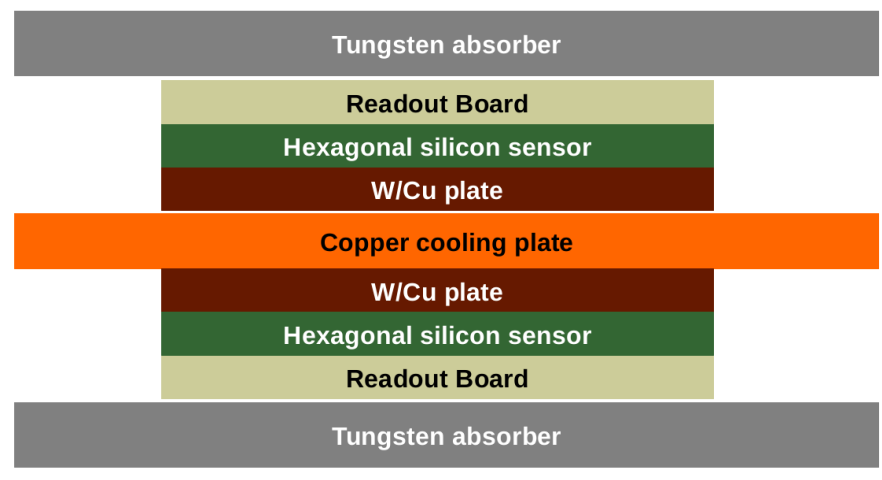

Figure 1: Assembly of modules on the copper cooling plate. board, where two 64-channel SKIROC2 ASICS [4] were used to readout the detector cells. The thickness of a module depended on the thickness of the W-Cu plate and was between 5 and $6 \mathrm{~mm}$.

Detector modules were mounted on either side of a $6 \mathrm{~mm}$ thick copper cooling plate, see Fig. 1. The mechanical support of the detector was a "hanging file" system for flexibility. In it the copper plates with modules attached were mounted interspersed with tungsten absorber plates to form the calorimeter stack. The thicknesses of the $\mathrm{W} / \mathrm{Cu}$ and tungsten plates were chosen so that the detector modules were at depths in radiation lengths of $0.6,1.4,2.0,2.8,3.4,4.3,5.1,6.1$,

\footnotetext{
${ }^{1}$ Hamamatsu Photonics, Hamamatsu, Japan
} 
$6.9,7.9,8.7,10.1,11.3,12.7,13.9$ and $15.3 \mathrm{X}_{0}$, for a normally incident particle. In the stack the distance between the silicon sensor and the tungsten absorber was set by the height of the readout boards at $\sim 5 \mathrm{~mm}$.

The DAQ was designed to be scaleable, using commercially available components mounted on custom PCBs. In the DAQ each sensor module was connected to a data collection FPGA, where data were collected during a beam spill and stored. Between the beam spills the data were transferred to an AVNET ZedBoard ${ }^{2}$ that communicated, via ethernet, to a control PC.

\section{Results}

Protons with an energy of $120 \mathrm{GeV}$ were used to calibrate the detector. The signal was modeled with a Gaussian convoluted with a Landau distribution, Fig. 2a, and compared with the response predicted by simulation for minimum ionizing particles (MIPs), to obtain the ADC $\rightarrow$ MIP conversion factor for each of the 16 sensors. The signal-to-noise ratio for a single MIP, observed across the channels was around 7 for all sensors. The signals in all cells above a threshold of 2 MIPs were used to find the distribution of the deposited energy in each silicon sensor. Fig. 2b shows the mean values of the deposited energy distributions as a function of the depth, for different beam energies. Fig. 3 is a typical $32 \mathrm{GeV}$ electron event display, demonstrating how the high granularity of the calorimeter enables the tracking of the shower development in both the transverse and longitudinal directions.

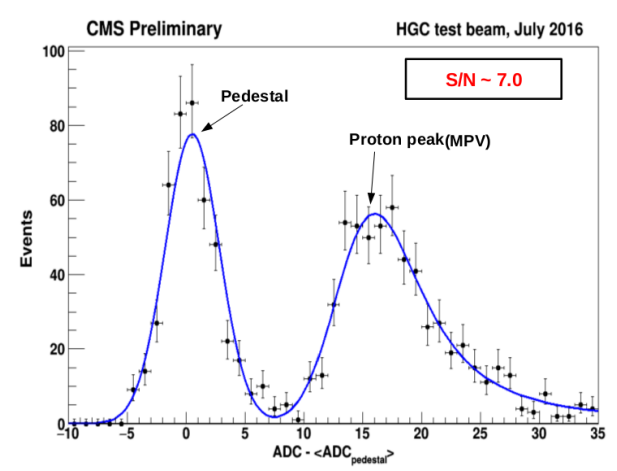

(a)

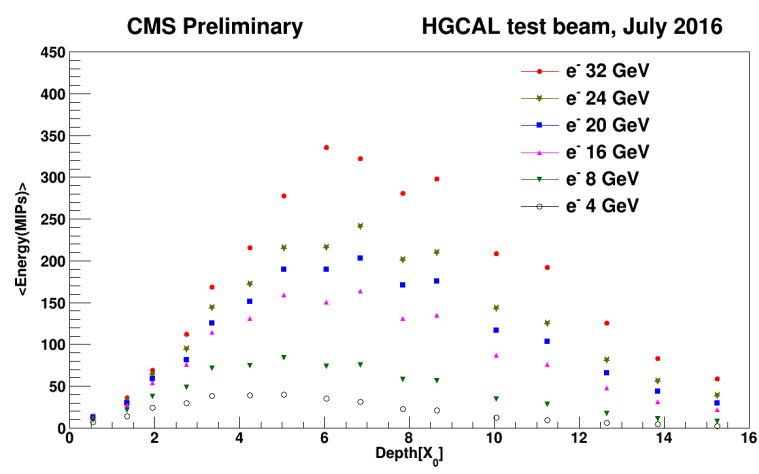

(b)

Figure 2: (a) Response to $120 \mathrm{GeV}$ protons in one detector channel. This signal was used for calibration. (b) Longitudinal shower profile of electrons at energies from 4-32 GeV. The shower maximum was reached at around the $9^{\text {th }}$ layer $\left(6.9 X_{0}\right)$. The tail of the shower was not observed as the total depth was only $15.3 X_{0}$.

The response of the detector to electrons was measured using the same procedure as above, with the energy deposited in each layer summed in order to obtain the total energy spectrum. A Gaussian was fitted to the core of this distribution. Fig. 4 shows the fitted mean as a function of the beam energy.

After these tests, eight detector modules were transported from Fermilab to CERN. Data were collected in two different detector configurations with electrons at energies ranging from 20-250

\footnotetext{
${ }^{2} \mathrm{http}: / /$ zedboard.org
} 


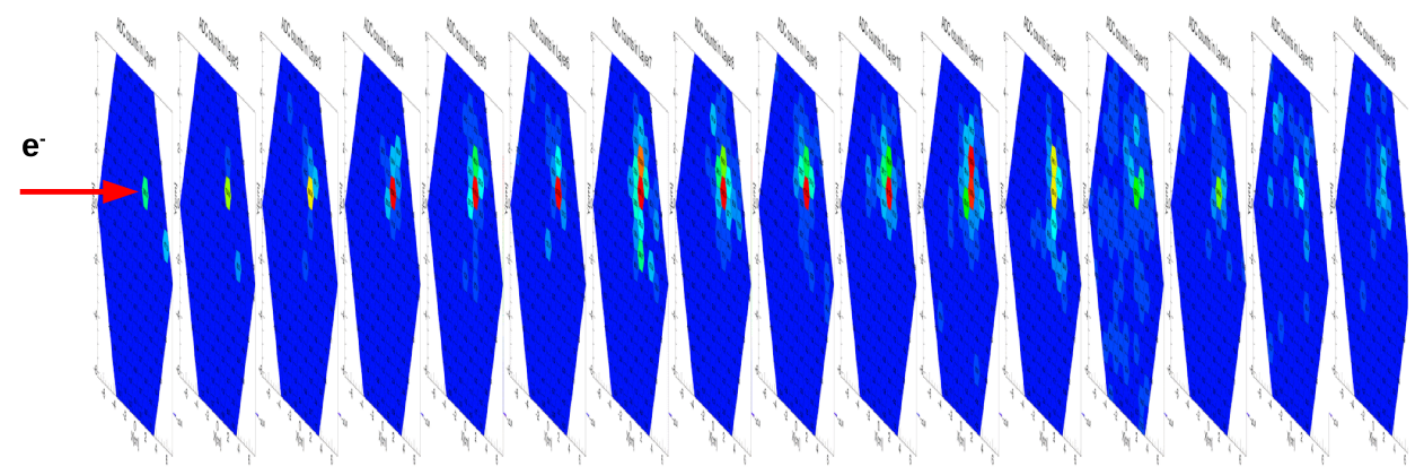

Figure 3: A $32 \mathrm{GeV}$ electron in the detector stack.

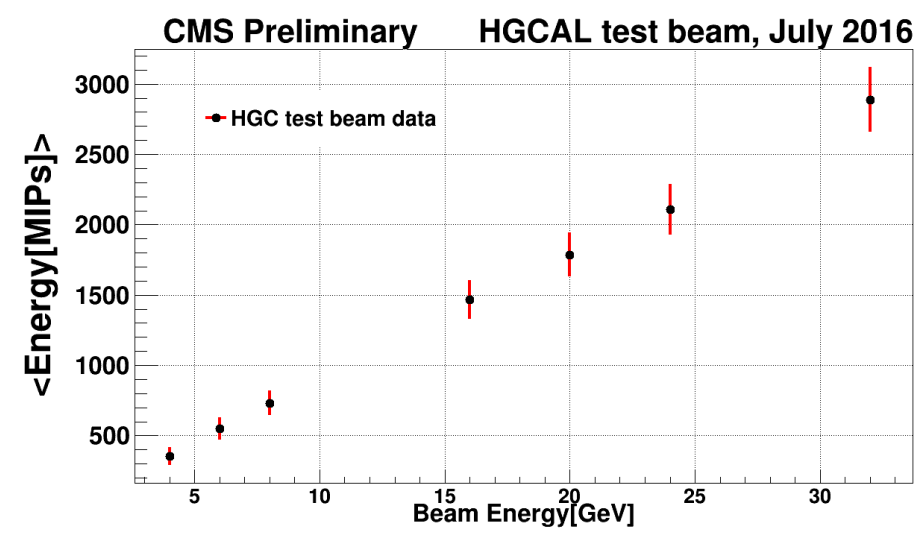

Figure 4: Electron response of the detector vs beam energy.

$\mathrm{GeV}$. The prototype has been operational since then at CERN collecting muon data, to study the uniformity of the detector. The analyses of both sets of test beam data and comparisons with a detailed simulation are still in progress.

\section{References}

[1] J. Butler, D. Contardo, M. Klute, J. Mans, and L. Silvestris eds. Technical Proposal for the Phase-II Upgrade of the CMS Detector.CMS Collaboration. CERN-LHCC-2015-010, LHCC-P-008. (June 2015).

[2] E. Curras et al. Radiation hardness and precision timing study of silicon detectors for the CMS High Granularity Calorimeter (HGC). AIDA-2020-PUB-2016-004, (May, 2016), Nuclear Instruments and Methods in Physics Research Section A, http://dx.doi.org/10.1016/j.nima.2016.05.008

[3] J.C. Brient and H. Videau, "The Calorimetry at the future e+ $e$ - linear collider", eConf $\mathbf{C 0 1 0 6 3 0}$ (2001) E3047, arXiv:hep-ex/0202004 [hep-ex].

[4] S. Callier, F. Dulucq, C. de La Taille, G. Martin-Chassard, and N. Seguin-Morea, "SKIROC2, front end chip designed to readout the Electromagnetic Calorimeter at the ILC", JINST 6 (2011) C12040. 